CASE REPORT

\title{
Spontaneous Coronary Artery Dissection and the Takotsubo Syndrome: An Association to be Taken into Account
}

\author{
Verónica Hernández Jiménez ${ }^{1 *}$, Aránzazu García Romero ${ }^{2}$ and Jesús Saavedra Falero ${ }^{3}$ \\ ${ }^{1}$ Cardiology Department, Infanta Elena Hospital, Valdemoro, Madrid, Spain \\ ${ }^{2}$ Cardiology Department, Infanta Elena Hospital, Valdemoro, Madrid, Spain \\ ${ }^{3}$ Cardiology Department, University Hospital of Getafe, Madrid, Spain
}

*Corresponding author: Verónica Hernández Jiménez, Cardiology Department, Infanta Elena Hospital, Avenida de los Reyes Católicos, 21, 28342 Valdemoro, Madrid, Spain, Tel: +34-918-94-84-10

\section{Keywords}

Spontaneous coronary artery dissection, Takotsubo syndrome, Coronariography, Myocardial infarction

\section{Introduction}

Spontaneous coronary artery dissection (SCAD) and Takotsubo syndrome (TTS) are two causes of acute non-arteriosclerotic myocardial infarction. These two entities share some characteristics such as greater involvement in women, preceded by an emotional stressor and an etiopathogeny not clearly known. Recently it has been seen that these two entities may be related. We describe a case of a young woman with a myocardial infarction due to a SCAD who developed a TTS.

\section{Case Report}

A 44-year-old woman, without known cardiovascular risk factors, who went to the Emergency Department due to a chest pain lasting 2 hours. She reported that she had episodes of chest pain of less intensity and duration in the last week, attributed to the stress and anxiety due to she was divorcing. The patient was not in the peri-partum period during presentation and she had not menopausal symptoms. The physical examination was anodyne, the electrocardiogram revealed a sinus tachycardia at 100 beats per minute, ST-elevation in leads II, III, AVF and V4-V6. Her laboratory values were normal except to an elevation of Troponin I of $9.7 \mathrm{ng} / \mathrm{ml}$ (normal < 0.01 ) and creatine kinase of $432 \mathrm{ng} / \mathrm{ml}$ (normal $<155)$. An urgent coronary angiography showed a severe stenosis in the distal segment of the intermediate artery with angiographic aspect of dissection, the remaining coronary arteries were normal (Figure 1).

Ventriculography showed hypokinesia of all the middle and apical segments of the left ventricle (LV) (Figure 2). The chest pain disappeared with the administration of nitroglycerin and there was regress

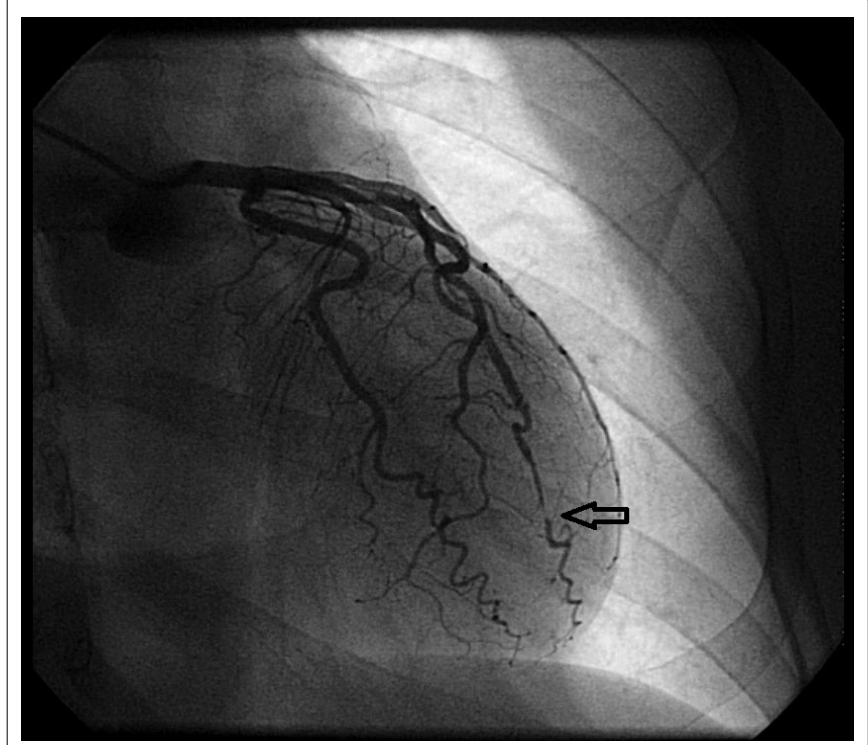

Figure 1: Coronary angiography in the right anterior oblique projection shows the dissection image in the distal segment of the intermediate artery (arrow).

Citation: Jiménez VH, Romero AG, Falero JS (2019) Spontaneous Coronary Artery Dissection and the Takotsubo Syndrome: An Association to be Taken into Account. Int J Clin Cardiol 6:135. doi. org/10.23937/2378-2951/1410135

Accepted: January 14, 2019; Published: January 16, 2019

Copyright: (c) 2019 Jiménez VH, et al. This is an open-access article distributed under the terms of the Creative Commons Attribution License, which permits unrestricted use, distribution, and reproduction in any medium, provided the original author and source are credited. 


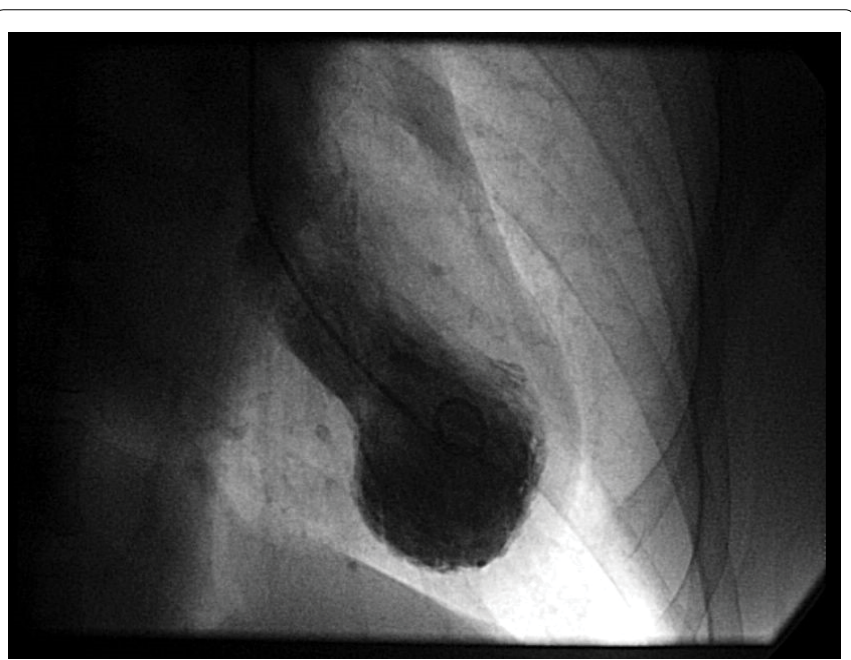

Figure 2: Left ventriculography shows systolic mid-apical left ventricular ballooning.

of the ST-elevations. Given the coronary anatomy and the clinical stability, it was managed conservatively with medical treatment with dual antiplatelet therapy and beta-blockers. The transthoracic echocardiogram showed severe hypokinesia in the middle and apical regions of $\mathrm{LV}$ beyond the territory corresponding to the intermediate artery and hyperkinesis in the basal segments, findings characteristic of TTS. Evolutively, the electrocardiogram developed wide spread T-waves inversión and a prolongation of the corrected QT interval (490 milliseconds). The study of connective tissue abnormalities was normal.

Follow-up echocardiogram (20 days after presentation) revealed a hypokinesia of the distal lateral segment of the LV. Cardiac magnetic resonance imaging to 2 months after the illness confirmed the normalization of LV segmental contractility and showed late gadolinium enhancement in the distal part of the LV lateral wall corresponding to the infarction of the intermediate artery.

The patient was included in cardiac rehabilitation program. She followed an interval training modality during 2 months without any problem. She remains asymptomatic.

\section{Discussion}

SCAD and TTS are two causes of acute non-atherosclerotic myocardial infarction [1]. Both conditions share certain characteristics: Affect predominantly woman, present ischemic electrocardiographic abnormalities, they are usually triggered by a stress emotion or less frequently by physical stress and the pathophysiological mechanism is not clear [2]. In both, coronary angiography is fundamental. The diagnosis of TTS includes the absence of images of plaque rupture or obstructive coronary disease [3] and transient apical hypokinesia is characteristic. On the other hand, the angiographic image of the SCAD is characteristic with the appearance of a radiolucent lumen and the contrast staining of arterial wall although sometimes more advanced imaging tech- niques such as IVUS are needed for confirmation [4]. One of the main differences between these two entities is that regional wall motion abnormalities in TTS extend beyond a single epicardial vascular territory [1].

Traditionally, SCAD and TTS have been considered as independent causes of myocardial infarction but recently some cases have been published where SCAD and TTS coexist [2,5-8]. According to the published reports, two hypotheses are proposed: 1) The SCAD produces a stressful situation with an excessive activation of the sympathetic system that triggers the TTS or 2) The external torsion force on the coronary arteries associated with wall motion abnormalities (segmental basal hyperkinesis) in TTS lead to a SCAD, especially in the area between the hyperkinesis segment and the hypokinesis segment $[1,5]$.

The optimal medical management of SCAD is not clear in part due to the limited clinical experience. A wide range of approaches, including conservative management, emergency revascularization with percutaneous coronary intervention or coronary artery bypass grafting have been reported. Patients presenting with acute myocardial infarction who have symptoms of ongoing ischemia or hemodynamic compromise should be considered for revascularization with $\mathrm{PCl}$ or coronary artery bypass grafting. A conservative therapy is the preferred strategy in stable patients because of a revascularization in patients with SCAD is technically challenging and associated with higher failure rates or complicaciones [9].

According to studies published, it is recommended long-term aspirin, beta blocker, and one year of clopidogrel, with the addition of a statin in patients with dyslipidemia. The use of beta blocker was associated with lower risk of recurrent SCAD [10]. Ongoing prospective studies on SCAD should further elucidate the medical management of this challenging and relatively unexplored condition. In TTS, the patients are managed with aspirin, beta blocker and angiotensinconverting enzyme inhibitors and diuretics as required for LV systolic dysfunction and volume overload.

The coexistence of these two entities may imply a worse prognosis with higher risk of complications such as acute heart failure or arrhythmias [6], for that reason it is necessary to correctly identify them for a better risk stratification.

In our case and taking into account the evolution of the tests, we believe that our patient suffered an acute myocardial infarction due to a SCAD in a distal segment of a non-main artery and that ischemia and stress due to pain and hospital admission triggered transient apical hypokinesia characteristic of TTS confirmed by the findings of ventriculography, echocardiography, and cardiac resonance, although it is difficult to confirm what occurred first. 
More studies are needed to know the clinical relevance and the prognostic implications of this association.

\section{Ethical Responsibility}

The publication of the article was valued and accepted by the ethical committee of our hospital.

\section{Conflicts of Interest}

There are no conflicts of interest.

\section{Acknowledgment}

Acknowledgments to the Hemodynamics Department of the Infanta Elena Hospital.

\section{References}

1. Buccheri D, Zambelli G (2017) The link between spontaneous coronary artery dissection and takotsubo cardiomyopathy: Análisis of the published cases. J Thorac Dis 9: $5489-5492$.

2. $Y$ Hassan $S$, Bohm $F$ (2016) The casual link between spontaneous coronary artery dissection coronary and takotsubo syndrome: A case presented with both conditions. Int J Cardiol 203: 828-831.

3. Madhavan M, Prasad A (2010) Proposed Mayo Clinic criteria for the diagnosis of Tako-Tusbo cardiomyopathy and long-term prognosis. Herz 35: 240-243.
4. Saw J, Mancini GB, Humphries KH (2016) Contemporary review on spontaneous coronary artery dissection. J Am Coll Cardiol 68: 297-312.

5. Chou AY, Sedlak T, Aymong E, Sheth T, Starovoytov A, et al. (2015) Spontaneous coronary artery dissection misdiagnosed as Takotsubo cardiomyopathy: A case series. Can J Cardiol 31: 1073.

6. Yalta K, Ucar F, Yilmaztepe M, Ozkalayci F (2016) Takotsubo cardiomyopathy and spontaneous coronary artery dissection: A subtle association with prognostic implications? Int J Cardiol 202: 174-176.

7. Y-Hassan S, Henareh L (2013) Spontaneous coronary artery dissection triggered post-ischemic myocardial stunning and takotsubo syndrome: Two different names for the same condition. Cardivasc Revasc Med 14: 109-112.

8. Y-Hassan S (2018) Spontaneous coronary artery dissection and takotsubo syndrome: An often overlooked association; review. Cardivasc Revasc Med 19: 717-719.

9. Saw J, Aymong E, Sedlak T, Buller CE, Starovoytov A, et al. (2014) Spontaneous Coronary artery dissection: association with predisposing arteriopathies and precipitating stressor and cardiovascular outcomes. Circ Cardiovasc Inter 7: 645655.

10. Saw J, Humphries K, Aymong E, Sedlak T, Prakash R, et al. (2017) Spontaneous Coronary artery dissection: Clinical Outcomes and Risk of recurrence. J Am Coll Cardiol 70: 1148-1158. 